\title{
Ulrich Felgner: Philosophie der Mathematik in der Antike und in der Neuzeit
}

\author{
Springer Spektrum 2020, XIX + 229 Seiten, \\ ISBN: 978-3-030-35933-1, €79,99; eBook \\ ISBN 978-3-030-35934-8, €62,92
}

\section{Rainer Löwen}

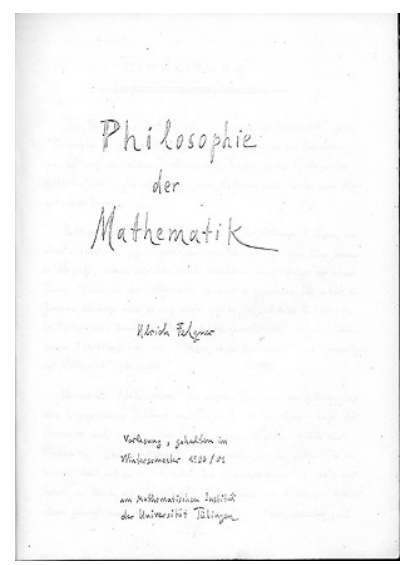

Die Antworten auf diese Fragen mögen heute vielen von uns als Selbstverständlichkeiten erscheinen: Es ist prinzipiell unmöglich zu sagen, was ein Punkt, eine Gerade, eine Zahl etc. ist. Man kann nur komplette Systeme von Punkten und Geraden oder von Zahlen definieren, und wesentlich ist nicht, was diese Objekte wirklich sind, sondern nur, was ihre gegenseitigen Beziehungen sind. Diese sind in Axiomen festgelegt, und als wahr gilt eine Aussage dann, wenn sie sich nach vereinbarten Regeln der Logik aus den Axiomen ableiten läßt. Bei dieser Antwort verliert man aber leicht aus den Augen, wie schwer erkämpft dieser heutige Standpunkt ist, und wel-

Rainer Löwen $(\bowtie)$

Technische Universität Braunschweig, Braunschweig, Deutschland

E-Mail: r.loewen@tu-bs.de
Zu den Schätzen meines Bücherschranks zählt ein in wunderbarer Kalligraphie handgeschriebenes Vorlesungsskript des Autors aus dem Jahr 1981, das er mir damals geschenkt hat, und mit dem ich mich einmal intensiver befaßt habe, wie die vielen Anmerkungen auf dem Umschlag bezeugen. Die Titelseite ist hier wiedergegeben.

Das vorliegende Buch mit dem gleichen Titel hat inhaltlich eher wenig Überschneidung mit dem damaligen Skript. Es konzentriert sich stärker auf einige grundlegende Fragestellungen, die man in wenigen Sätzen so zusammenfassen kann: Was sind eigentlich die Gegenstände der Mathematik? Existieren sie unabhängig von uns? Von welcher Art ist ihre Existenz? Und was gibt uns die Gewißheit, wahre Aussagen über sie machen zu können? 
che Fallen des Denkens zu überwinden waren, um dahin zu gelangen. Das Anliegen des Buches besteht darin, ein tieferes Verständnis der aktuellen Denkweise zu vermitteln, indem der historische Weg in allen Stationen nachgezeichnet wird, der von den Anfängen der erkenntnisorientierten Mathematik im antiken Griechenland bis zu Hilbert und Gödel führt. Dazu ist es notwendig, auch das geistige Umfeld der jeweils betrachteten Zeit zu beleuchten und vor diesem Hintergrund zu untersuchen, warum von bestimmten Grundlagenfragen der Mathematik eine solch starke Beunruhigung ausging und warum die heute so offensichtlich scheinenden Auswege es keineswegs immer waren. Dies führt uns der Autor an Hand zahlreicher Quellen plastisch vor Augen. Zugleich geht er detailliert auf mathematische Entdeckungen und Entwicklungen ein, die einen starken Einfluss auf die Diskussion der Grundlagenprobleme hatten. Hierzu zählen beispielsweise die Entdeckung der Inkommensurabilität, der Beweis der Existenz unendlich vieler Primzahlen, generell das Phänomen des Unendlichen in der Mathematik, ebenso nichtkonstruktive Existenzbeweise wie im Fall von Hilberts Basissatz. Eine große Rolle spielen auch Paradoxien wie das Zenonsche Paradox von Achilles und der Schidkröte und die Russelsche Antinomie in der Mengenlehre, die die Gefahren bei ungezügelter Verwendung des Mengenbegriffs deutlich macht.

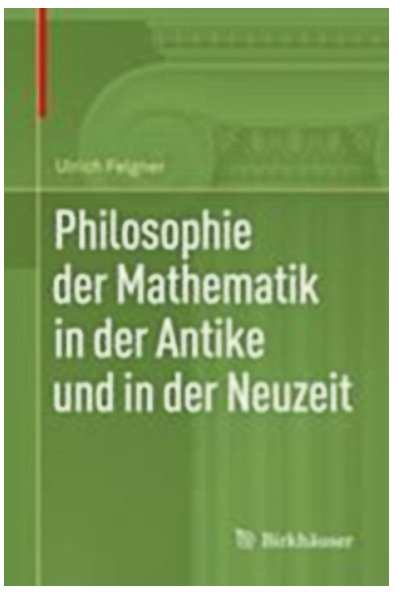

Beeindruckend ist für mich, welch ungeheure Fülle von ganz unterschiedlichen Ansätzen und Positionen zu den Grundlagenfragen es im Lauf von zweitausend Jahren gegeben hat. Manches davon war mir von Ferne bekannt, vieles auch gänzlich unbekannt. Der Autor schöpft aus einem gewaltigen Reservoir an Wissen über sein Thema. Das meiste davon sind Dinge, die im normalen Mathematikerleben gar nicht vorkommen, und gewiss nicht im mathematischen Alltag. Mit ihnen bekannt gemacht $\mathrm{zu}$ werden fand ich überaus bereichernd. Es ist mir nicht möglich, diese Vielfalt von Sichtweisen, die das Buch in 20 Kapiteln mit über 100 Abschnitten ausführlich beleuchtet, auch nur ansatzweise zu beschreiben. Die Beiträge, die eine Person, eine Strömung oder eine Epoche zu den Grundlagenproblemen der Mathematik geleistet hat, werden jeweils mit sorgfältig ausgewählten Originalzitaten in der Originalsprache und in deutscher Übersetzung vorgestellt und ihre ideengeschichtliche Wirkung wird gründlich diskutiert. Jedes Kapitel enthält ein eigenes Literaturverzeichnis mit Original- und Sekundärschriften.

Die hier besprochenen Fragen haben keineswegs nur Mathematiker bewegt, sondern auch Philosophen (Platon, Aristoteles, Locke, Hobbes, Kant, ...) und Theologen (Augustinus und andere Kirchenväter). Man gewinnt sogar den Eindruck, dass die Philosophen den Verlauf der Entwicklung noch stärker beeinflusst haben als die Mathematiker. Das gilt sicherlich für die Wissenschaftstheorie des Aristoteles, der von einer Wissenschaft als erstes verlangt, dass sie einen klar bestimmten Gegenstand hat. Das Ringen um den Gegenstand der Mathematik war daher immer auch ein Ringen um ihren Wissenschaftsstatus. Bei Kant verhält es sich dagegen eher so, dass 
seine Auffassung, dass die Anschauung einen Platz in der mathematischen Wahrheitsfindung habe, die Mathematiker zum Widerspruch gereizt hat, ihnen gleichzeitig aber auch klar gemacht hat, dass sie größere Anstrengungen unternehmen mussten, um das Fundament der Mathematik solider zu gestalten. Er wurde damit zum Auslöser der Entwicklung, die schießlich zu Hilberts Auffassung von Axiomatik führt.

In einem (für das Thema des Buches eher nebensächlichen) Punkt möchte ich dem Autor widersprechen, nämlich bei der Bewertung der berühmten Zenonschen Paradoxien, etwa der von Achilles und der Schildkröte. Zenons Argumente werden vom Autor als fehlerhaft eingestuft. Die recht knappe Begründung lautet, hier würden zwei verzahnte Grenzprozesse (in Zeit und Raum) unzulässigerweise entkoppelt. Das kann ich so nicht erkennen. Tatsächlich konstruiert doch Zenon zwei Folgen, nämlich eine von Punkten auf der Rennstrecke und eine von zugehörigen Zeitpunkten, die beide in der jeweiligen Anordnung streng monoton wachsend und dennoch nach oben beschränkt sind. Dies ist in meinen Augen die Paradoxie, und diese Paradoxie besteht heute genauso wie vor zweitausend Jahren. Was Zenon damit offenbart ist die grundsätzliche Schwierigkeit, die uns das Verständnis unendlicher (und hier auch angeordneter) Mengen macht, weil es uns so schwer fällt, uns von unserer Erfahrung mit endlichen Anordnungen zu lösen. Die Einkleidung und die Kopplung der beiden Folgen durch die Betrachtung eines Bewegungsvorgangs dient einerseits als Mittel zur Konstruktion beider Folgen und andererseits als ein Mittel der Veranschaulichung. Zenon wird selbst nicht geglaubt haben, er hätte die Unmöglichkeit von Bewegung bewiesen, auch wenn er das so formuliert hat.

Nach der Schilderung des langen Wegs der Erkenntnis, die das Buch uns gibt, stelle ich mir gern diesen Weg als Kurve in einem metrischen Raum vor. Dann scheint mir die Distanz von Euklid zu Dedekind und Hilbert in diesem Raum wesentlich geringer zu sein als die längs der Kurve gemessene Distanz in der ,,inneren Metrik“. Euklid stellt zwar Definitionen für Punkte und Geraden an den Anfang seiner Geometrie, um einen oberflächlichen Bezug zur Anschauung herzustellen, aber aus diesen Definitionen lässt sich nichts ableiten und mit ihnen wird nie gearbeitet. Er arbeitet nur mit den Axiomen, die die gegenseitigen Beziehungen von Punkten und Geraden regeln. Daher teile ich übrigens nicht die Auffassung des Autors, der auf Seite 50 diese Definitionen im Aufbau von Euklids Theorie für unverzichtbar erklärt. Bei Euklid sehe ich im Keim den Standpunkt von Hilbert bereits vorgedacht. Aber unter dem Einfluss von Aristoteles konnte dieser Keim sich nicht entfalten, und erst Kant hat, eher unbeabsichtigt, den Anstoß dazu gegeben, dass der Weg von Euklid konsequent zuende geführt werden konnte. Dies ist freilich meine persönliche, naive und stark verkürzte Deutung. Das Buch weicht der Komplexität des Geschehens nicht aus, wie ich es hier tue, und wer diese spannende Geschichte im einzelnen verstehen will, kommt um die Lektüre nicht herum.

Das Buch ist sehr angenehm zu lesen, wenn man sich erst einmal an den recht freigiebigen Gebrauch von Kursivsatz, Anführungszeichen und Apostrophen gewöhnt hat, der zu einem leicht barocken Erscheinungsbild führt. In weiten Teilen setzt das Buch sehr wenig mathematische Vorkenntnisse vorus. Wo irgend möglich, werden mathematische Begriffe und Sachverhalte sorgfältig und verständlich erklärt. Es wird die gesamte Mathematik berücksichtigt. Das eigene Arbeitsgebiet des Autors (die mathematische Logik) spielt naturgemäß eine wichtige Rolle (etwa im Zusammen- 
hang mit den Sätzen von Gödel über die Grenzen mathematischer Erkenntnis), wird aber nicht besonders herausgehoben. So wird dieses Buch nach meiner Einschätzung für viele ein großer Gewinn sein, angefangen von interessierten Nichtmathematikern, die schon immer wissen wollten, wovon Mathematik eigentlich handelt, bis hin zu Fachmathematikern, die gelegentlich beunruhigt sind angesichts der Schwierigkeit, das Vorgehen der Mathematik auf eine sichere Grundlage zu stellen, oder die sich nur ihres Verständnisses dieser Grundlage vergewissern wollen. Dem Autor gebührt großer Dank dafür, und ich empfehle die Lektüre wärmstens.

Funding Open Access funding enabled and organized by Projekt DEAL.

Open Access Dieser Artikel wird unter der Creative Commons Namensnennung 4.0 International Lizenz veröffentlicht, welche die Nutzung, Vervielfältigung, Bearbeitung, Verbreitung und Wiedergabe in jeglichem Medium und Format erlaubt, sofern Sie den/die ursprünglichen Autor(en) und die Quelle ordnungsgemäß nennen, einen Link zur Creative Commons Lizenz beifügen und angeben, ob Änderungen vorgenommen wurden.

Die in diesem Artikel enthaltenen Bilder und sonstiges Drittmaterial unterliegen ebenfalls der genannten Creative Commons Lizenz, sofern sich aus der Abbildungslegende nichts anderes ergibt. Sofern das betreffende Material nicht unter der genannten Creative Commons Lizenz steht und die betreffende Handlung nicht nach gesetzlichen Vorschriften erlaubt ist, ist für die oben aufgeführten Weiterverwendungen des Materials die Einwilligung des jeweiligen Rechteinhabers einzuholen.

Weitere Details zur Lizenz entnehmen Sie bitte der Lizenzinformation auf http://creativecommons.org/ licenses/by/4.0/deed.de. 